\title{
Fully integrated receiver for free-space visible light communication
}

\author{
Dong Yan $^{1}$, Xurui Mao ${ }^{2 a)}$, Jia Cong ${ }^{1}$, and Sheng $\mathrm{Xie}^{3}$
}

Abstract A fully integrated visible light communication (VLC) receiver is presented in this paper. The proposed VLC receiver chip is designed for free-space communication. Utilizing a laser diode as light source, the measured $-3 \mathrm{~dB}$ bandwidth of receiver is $420 \mathrm{MHz}$ over $0.5 \mathrm{~m}$ distance without lens. The integrated receiver is manufactured in an UMC $0.18 \mu \mathrm{m}$ CMOS process, and the chip area is $889 \times 570 \mu \mathrm{m}^{2}$. This receiver chip can be used in VLC systems with the following advantages: suitable for VLC free-space channel, low requirements for focusing and alignment, low cost, low power-consumption, compatible with standard CMOS technology, and high integration-density.

Keywords: visible light communication (VLC), receiver, avalanche photodiode (APD), free-space, CMOS

Classification: Integrated optoelectronics

\section{Introduction}

Simultaneously offering data communication and indoor illumination make VLC gain plenty of attention in the past few years $[1,2,3,4,5,6,7,8,9,10,11,12,13]$. In the future, VLC has the potential to play a major part in smart home and next generation communication networks. It is a significant work to increase the achievable data rates and make it can be integrated within existing network infrastructures. The future of VLC systems depends on the ability to fabricate low cost transceiver components and to realize the promise of high data rates. Integrated high-speed solutions for the VLC source driver and receiver are required $[14,15,16,17,18,19,20,21,22,23$, $24,25,26]$.

A research team in Japan proposed a VLC system based on phosphor LED transmitter and a CMOS camera receiver. Using on-off keying (OOK) modulation, the datarate is $10-20 \mathrm{Mbps}$ [27]. Another $24 \mathrm{Mbps}$ VLC system based on ordinary phosphor white LED without pre-emphasis, a commercial silicon PIN photodiode (PD) and CMOS receiver is also presented [28]. Due to the limited communication bandwidth of phosphorescent LED, the achievable data-rate of VLC system is not fast enough. Due to the long photoluminescence lifetimes of the phos-

\footnotetext{
${ }^{1}$ School of Electrical and Information Engineering, Tianjin University, Tianjin 300072, China

${ }^{2}$ State Key Laboratory on Integrated Optoelectronics, Institute of Semiconductors, Chinese Academy of Sciences, Beijing 100083, China

${ }^{3}$ School of Microelectronics, Tianjin University, Tianjin 300072, China

a) maoxurui@semi.ac.cn
}

DOI: 10.1587/elex.16.20190418

Received June 28, 2019

Accepted August 1, 2019

Publicized August 26, 2019

Copyedited September 25, 2019 phor, the communication bandwidths of phosphor LED is only a few MHz. This is the bottleneck for high speed data communications. Based on $\mu$ LED [14] and laser diode (LD) $[29,30]$, which have much better modulation bandwidth than LED, the data-rate of integrated VLC system can be improved.

For the existing optical receivers, the area of detector is small, for the purpose of achieving higher communication speed. However, the receiver with small detector is not suitable for free-space VLC, mainly due to the low optical power density and high requirements for focusing and alignment. Therefore, we proposed a fully integrated VLC receiver with much larger avalanche photodiode (APD), which is more suitable for free-space VLC. We also measured and analyzed the trade-off relationship between the area and frequency response of APD. Based on the study of APD, we proposed a fully integrated VLC receiver with active inductor and post-equalization for frequency compensation of the large area APD.

This paper is organized as follows: Section 2.1 presents the trade-off relationship between the area and frequency response of APD, Section 2.2 presents the design of receiver circuits. Experimental results are reported and discussed in Section 3. Finally, conclusions are drawn in Section 4.

\section{Design of the proposed VLC receiver}

\subsection{APD array}

In this section, we measured and analyzed the frequency response characteristics of APDs.

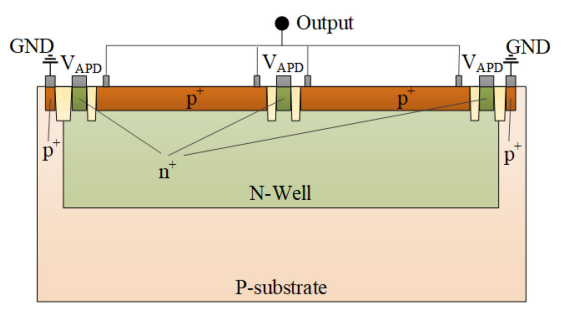

Fig. 1. Cross-section view of APD.

The structure of APD in this paper is shown in Fig. 1. Generally, there are mainly two kinds of APD structures for visible light detection. One is $\mathrm{P}+/ \mathrm{N}$-well junction, and the other is $\mathrm{N}$-well (or deep N-well)/P-substrate junction. The junction depth of $\mathrm{N}$-well/P-sub is much deeper than $\mathrm{P}+/ \mathrm{N}$-well. The response speed of $\mathrm{P}+/ \mathrm{N}$-well APD is faster than N-well/P-sub APD. Therefore, we utilized the $\mathrm{P}+/ \mathrm{N}$-well structure for the APD design. 


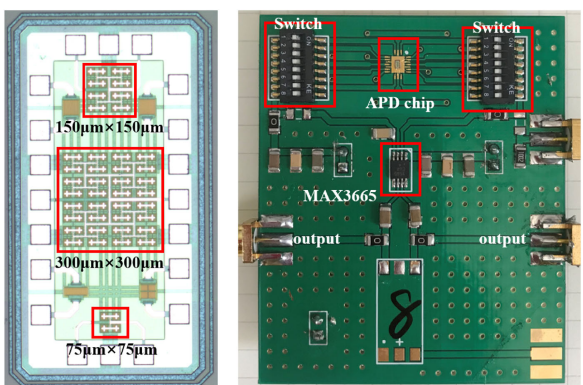

Fig. 2. The micrograph of APD array and the photo of DUT.

Three APDs of different size are fabricated in UMC (United Microelectronics Corporation) $0.18 \mu \mathrm{m}$ CMOS process, which is shown in Fig. 2. The dimensions of the three APDs are $75 \mu \mathrm{m} \times 75 \mu \mathrm{m}, 150 \mu \mathrm{m} \times 150 \mu \mathrm{m}$ and $300 \mu \mathrm{m} \times 300 \mu \mathrm{m}$, respectively. The connection wires from every APD unit to output electrode are designed to be the same, in order to minimize the influence of electrode connection wire. The APD chip is packaged on a PCB (Printed Circuit Board) using bonding wires as the DUT (Device Under Test). Because the output photocurrent of APD is too weak to be measured directly with a testing instrument of $50 \Omega$ port. Therefore, a high-speed transimpedance amplifier (MAX3665) with $8 \mathrm{k} \Omega$ gain is used for photocurrent amplification. The dimensions of the DUT are $5 \mathrm{~cm} \times 6 \mathrm{~cm}$. The DUT is shown in Fig. 2. The chip capacitors on the DUT are used to filter off the high-frequency components of the DC power supply.

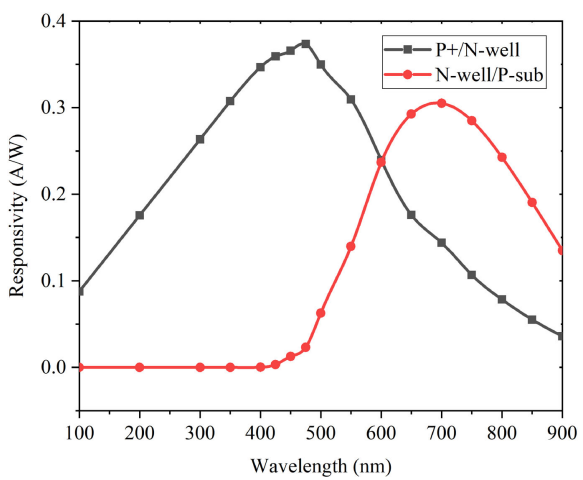

Fig. 3. Normalized responsivity against wavelength (simulation).

Fig. 3 shows the simulation result of APD response. The cross-sectional structure and material determined the shape of response curve. The responsivity peak of $\mathrm{P}+/$ $\mathrm{N}$-well junction is $450 \mathrm{~nm}$, and the responsivity peak of $\mathrm{N}$-well/P-sub junction is $750 \mathrm{~nm}$. For the APD structure proposed in this paper, we choose the $450 \mathrm{~nm}$ light source for measurement.

For big size APD, the photocurrent is large, which means better SNR (signal to noise ratio). However, the parasitic junction capacitance of big size APD has great influence on frequency response characteristics. Next, we measured and analyzed the relationship between the area and frequency response of APD.

A LD (OSRAM PL TB450B) is used as the light source for measurement. The $-3 \mathrm{~dB}$ bandwidth of $\mathrm{LD}$ is more than $1 \mathrm{GHz}$, which is enough for our experiment.

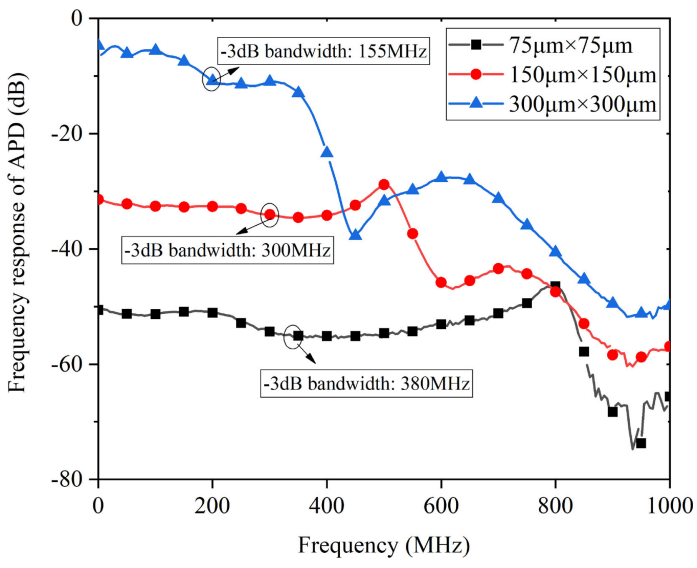

Fig. 4. Frequency response of APD.

The network analyzer (Agilent E5062A) is utilized for frequency response measurement.

Fig. 4 shows the experiment results of frequency response. For the low-frequency band, larger area means higher frequency response value. The start frequency response value of $300 \mu \mathrm{m} \times 300 \mu \mathrm{m}$ APD is $-5 \mathrm{~dB}$. The start frequency response value of $75 \mu \mathrm{m} \times 75 \mu \mathrm{m}$ APD is only $-50 \mathrm{~dB}$, which is very close to the background noise of free-space VLC (approximately $-60 \mathrm{~dB}$ ). Therefore, $75 \mu \mathrm{m} \times 75 \mu \mathrm{m}$ APD maybe not suitable for free-space VLC. The $-3 \mathrm{~dB}$ bandwidth of three APDs are $155 \mathrm{MHz}$, $300 \mathrm{MHz}$ and $380 \mathrm{MHz}$, respectively. Considering the response and bandwidth at the same time, we finally choose $150 \mu \mathrm{m} \times 150 \mu \mathrm{m}$ APD for the VLC receiver design.

\subsection{Design of the receiver circuits}

Utilizing the $150 \mu \mathrm{m} \times 150 \mu \mathrm{m}$ APD, we proposed a fully integrated VLC receiver with active inductor peaking and post-equalization.

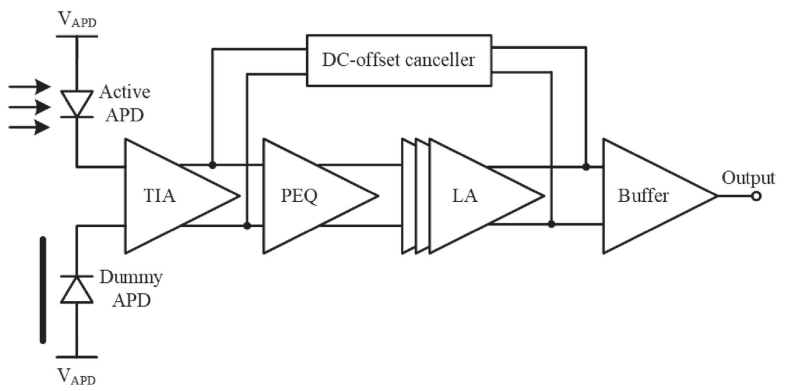

Fig. 5. The block diagram of VLC receiver.

Fig. 5 shows the block diagram of the proposed VLC receiver chip. The receiver is made up of APD, differential transimpedance amplifier (TIA), post-equalization (PEQ), limiting amplifier (LA) output buffer and DC-offset canceller. The dummy APD is covered by the top metal. The active APD is used to detect the visible light signal. Both the dummy APD and the active APD have the same size. The post-equalization is added to compensate for the bandwidth limitation of APD. Next, the detail of circuits is discussed.

The circuits of receiver are shown in Fig. 6. Active inductors are used instead of resistors as the drain loads, in order to improve the high frequency response. PEQ can de- 


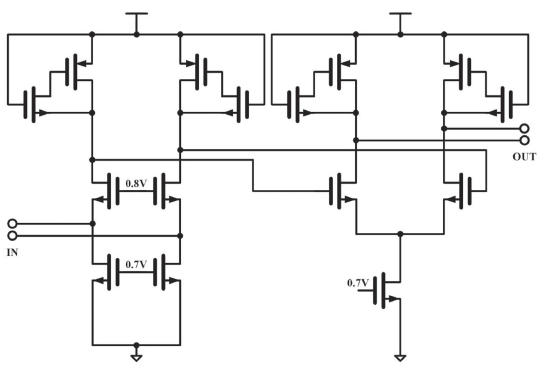

(a)

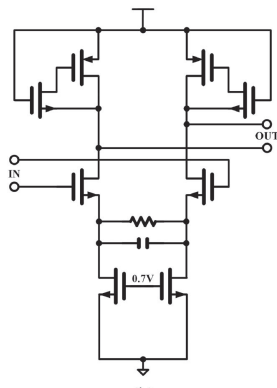

(b)

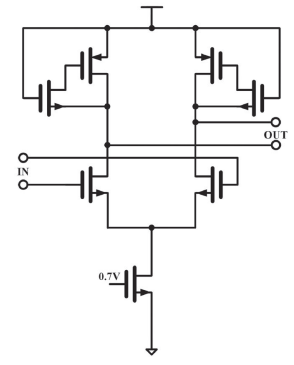

Fig. 6. Schematic of TIA, PEQ and LA.

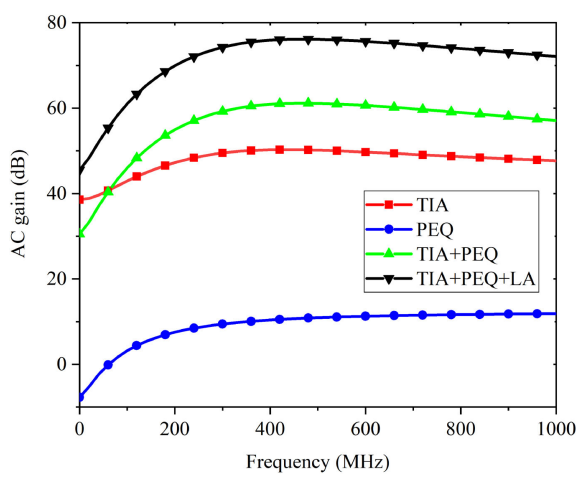

Fig. 7. AC gain simulation results.

crease the response in the low-frequency region but increase the amplitude of the signal in the high-frequency region.

Fig. 7 shows the ac gain simulation results. The TIA with active inductors compensated for $10 \mathrm{~dB}$ in the $0-300$ $\mathrm{MHz}$ band. The gain of PEQ is not more than $10 \mathrm{~dB}$. But the PEQ compensated for $20 \mathrm{~dB}$ in the $0-300 \mathrm{MHz}$ band. For the whole receiver, the peak ac gain value is $75 \mathrm{~dB}$ at $300 \mathrm{MHz}$. The reason why we designed $300 \mathrm{MHz}$ as the peak point is that the $-3 \mathrm{~dB}$ bandwidth of $150 \mu \mathrm{m} \times$ $150 \mu \mathrm{m}$ APD is $300 \mathrm{MHz}$. By this way, the VLC receiver can achieve better frequency compensation performance.

Fig. 8 shows the micrograph of the VLC receiver chip. The VLC receiver we propose was fabricated by UMC $0.18 \mu \mathrm{m}$ standard CMOS process, and the chip area is
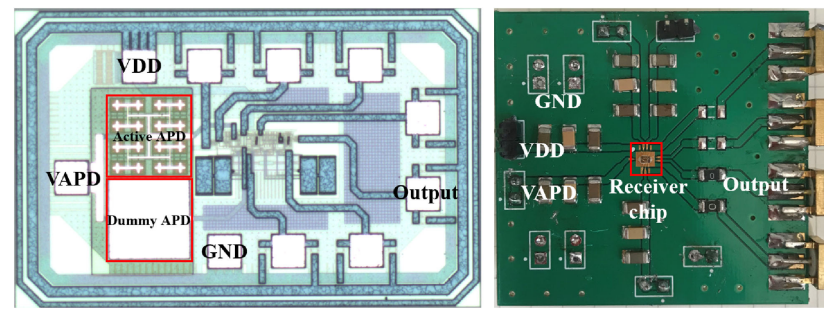

Fig. 8. The micrograph of VLC receiver and the photo of DUT.
$889 \times 570 \mu \mathrm{m}^{2}$. The DC power supply for the chip is $1.8 \mathrm{~V}$. All pads and some large components are marked in the figure. The VLC receiver chip is packaged on a PCB using bonding wires. The dimensions of the DUT are $4.5 \mathrm{~cm} \times 4.5 \mathrm{~cm}$. The experimental results are discussed in the next section.

\section{Experiment results}

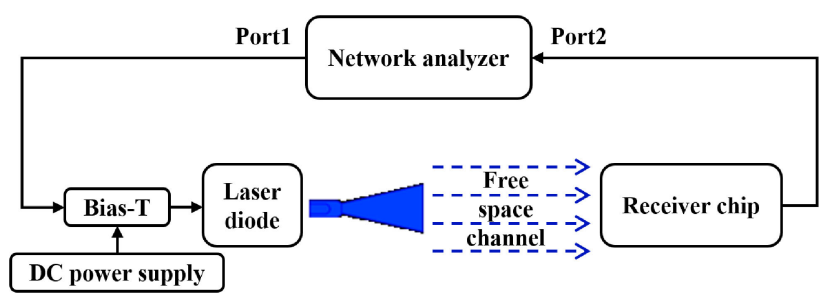

Fig. 9. Experimental setup to measure the frequency response of the receiver.

The experimental setup is shown in Fig. 9. A LD (OSRAM PL TB450B) is driven by the Bias-T (Mini-Circuits ZFBT$4 \mathrm{R} 2 \mathrm{GW}+$ ), the DC bias voltage of LD is $4 \mathrm{~V}$. We used a network analyzer (Agilent E5062A) to measure the frequency response of the receiver. Port 1 of the network analyzer was connected to Bias-T. The output of receiver was connected to port 2 of the network analyzer. The output-power of the network analyzer is $0 \mathrm{dBm}$.

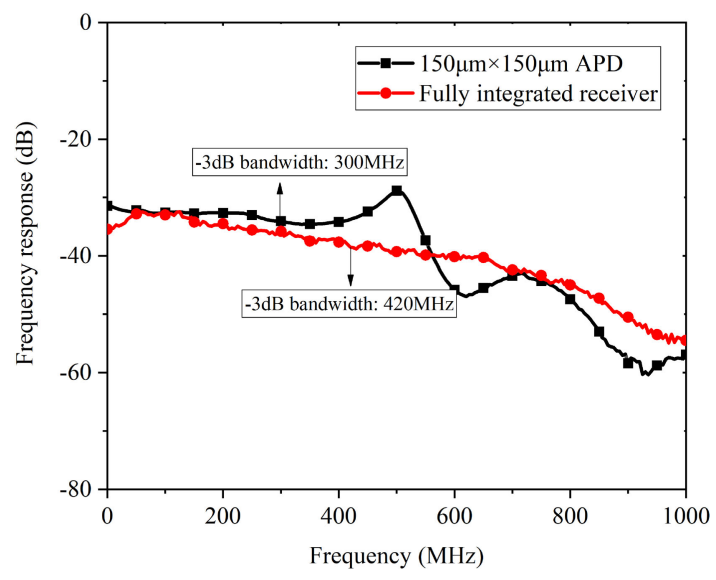

Fig. 10. Frequency response of the VLC receiver chip.

The experimental results of the frequency response are shown in Fig. 10. The black curve shows the frequency response of $150 \mu \mathrm{m} \times 150 \mu \mathrm{m}$ APD. The red curve shows the frequency response of VLC receiver chip. Since PEQ can decrease the response in the low-frequency region but increase the amplitude of the signal in the high-frequency region. The $-3 \mathrm{~dB}$ bandwidth of the $150 \mu \mathrm{m} \times 150 \mu \mathrm{m}$ APD could be extended from $300 \mathrm{MHz}$ to $420 \mathrm{MHz}$.

At last, the waveform of output signal is measured. The experimental setup is shown in Fig. 11. The RF signal generator (Agilent E4438C) is added to the RF port of Bias-T. The output-power of the RF signal generator is $0 \mathrm{dBm}$. The oscilloscope (RIGOL DS6104) is used to record the output waveform. 


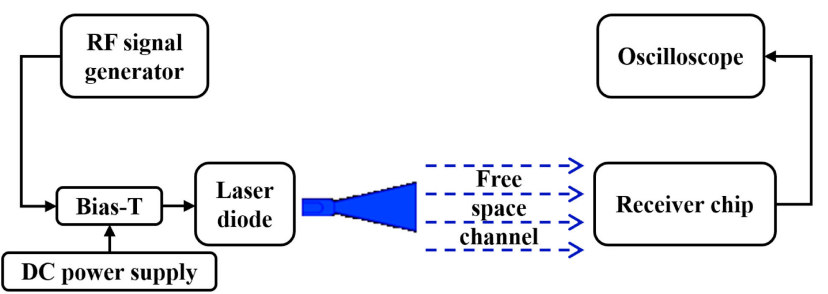

Fig. 11. Experimental setup to measure the waveform of the receiver.

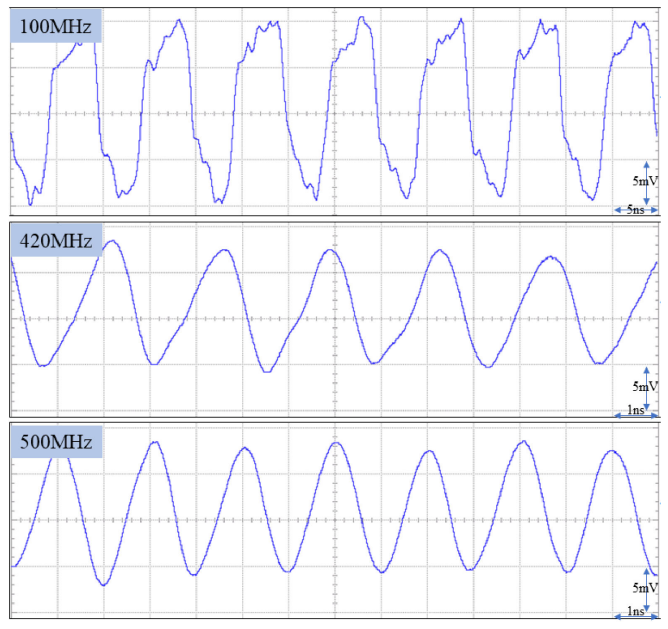

Fig. 12. Waveform of the VLC receiver output.

The output-frequency of the RF signal generator is changed from $100 \mathrm{MHz}$ to $500 \mathrm{MHz}$. The output waveform of different input frequency is shown in Fig. 12. Because of the free-space communication with no lens for focusing, the optical power is weak, the output signal voltage swing of receiver chip is relatively small. The peak to peak voltage of $100 \mathrm{MHz}$ waveform is $20.3 \mathrm{mV}$, and the peak to peak voltage of $420 \mathrm{MHz}$ waveform is $14.2 \mathrm{mV}$. The waveform of $420 \mathrm{MHz}$ is clear and stable, which means good communication performance under this frequency.

\section{Conclusion}

In this paper, the trade-off relationship between the area and frequency response of APD is analyzed. Based on the study of APD, we proposed a free-space VLC receiver with large area APD. Active inductor peaking and postequalization technique are used for frequency compensation of the large area APD. Utilizing a LD as the light source, the measured $-3 \mathrm{~dB}$ bandwidth of receiver chip is $420 \mathrm{MHz}$ over $0.5 \mathrm{~m}$ distance without lens. The VLC receiver we proposed is designed for free-space communication, and has the advantages of low cost, low powerconsumption, compatible with standard CMOS technology, and high integration-density.

\section{Acknowledgments}

This work was supported in part by the National Natural Science Foundation of China under Grant 61874104, in part by the National Key Research and Development Program of China under Grant 2017YFB0403603, and in part by the Open Fund of the State Key Laboratory on Integrated Optoelectronics under Grant IOSKL2017KF07.

\section{References}

[1] S. Rajagopal, et al.: "IEEE 802.15.7 visible light communication: Modulation schemes and dimming support," IEEE Commun. Mag. 50 (2012) 72 (DOI: 10.1109/MCOM.2012.6163585).

[2] A. Jovicic, et al.: "Visible light communication: Opportunities, challenges and the path to market," IEEE Commun. Mag. 51 (2013) 26 (DOI: 10.1109/MCOM.2013.6685754).

[3] J. Gancarz, et al.: "Impact of lighting requirements on VLC systems," IEEE Commun. Mag. 51 (2013) 34 (DOI: 10.1109/MCOM. 2013.6685755).

[4] J. Armstrong, et al.: "Visible light positioning: A roadmap for international standardization," IEEE Commun. Mag. 51 (2013) 68 (DOI: 10.1109/MCOM.2013.6685759).

[5] M. Ayyash, et al.: "Coexistence of WiFi and LiFi toward 5G: Concepts, opportunities, and challenges," IEEE Commun. Mag. 54 (2016) 64 (DOI: 10.1109/MCOM.2016.7402263).

[6] T. Yamazato and S. Haruyama: "Image sensor based visible light communication and its application to pose, position, and range estimations," IEICE Trans. Commun. E97-B (2014) 1759 (DOI: 10.1587/transcom.E97.B.1759).

[7] H. Li, et al.: "High bandwidth visible light communications based on a post-equalization circuit," IEEE Photon. Technol. Lett. 26 (2014) 119 (DOI: 10.1109/LPT.2013.2290026).

[8] Y. Wang, et al.: "8-Gb/s RGBY LED based WDM VLC system employing high-order CAP modulation and hybrid post equalizer," IEEE Photon. J. 7 (2015) 1 (DOI: 10.1109/JPHOT.2015.2489927).

[9] L. Zeng, et al.: "High data rate multiple input multiple output (MIMO) optical wireless communications using white LED lighting,” IEEE J. Sel. Areas Commun. 27 (2009) 1654 (DOI: 10.1109/ JSAC.2009.091215).

[10] $\mathrm{S}$. $\mathrm{Wu}$, et al.: "Visible light communications for $5 \mathrm{G}$ wireless networking systems: From fixed to mobile communications," IEEE Netw. 28 (2014) 41 (DOI: 10.1109/MNET.2014.6963803).

[11] P. H. Pathak, et al.: "Visible light communication, networking, and sensing: A survey, potential and challenges," IEEE Commun. Surveys Tuts. 17 (2015) 2047 (DOI: 10.1109/COMST.2015. 2476474).

[12] H. Haas, et al.: "What is LiFi?" J. Lightw. Technol. 34 (2015) 1533 (DOI: 10.1109/JLT.2015.2510021).

[13] L. U. Khan: "Visible light communication: Applications, architecture, standardization and research challenges," Digital Communications and Networks 3 (2017) 78 (DOI: 10.1016/j.dcan.2016. 07.004).

[14] S. Rajbhandari, et al.: "High-speed integrated visible light communication system: Device constraints and design considerations," IEEE J. Sel. Areas Commun. 33 (2015) 1750 (DOI: 10.1109/JSAC. 2015.2432551).

[15] S. Itoh, et al.: "A CMOS image sensor for $10 \mathrm{Mb} / \mathrm{s} 70 \mathrm{~m}$-range LED-based spatial optical communication,” ISSCC Dig. Tech. Papers (2010) 402 (DOI: 10.1109/ISSCC.2010.5433978).

[16] S. Zhang, et al:: "1.5 Gbit/s multi-channel visible light communications using CMOS-controlled GaN-based LEDs,” J. Lightw. Technol. 31 (2013) 1211 (DOI: 10.1109/JLT.2013.2246138).

[17] D. Yan, et al:: "Design fully integrated driver circuit for phosphorescent white light-emitting-diode high speed real-time wireless communication," IEEE Photon. J. 11 (2019) 1 (DOI: 10.1109/ JPHOT.2019.2904607).

[18] F. Che, et al:: "A fully integrated IEEE 802.15 .7 visible light communication transmitter with on-chip 8-W $85 \%$ efficiency boost LED driver,” J. Lightw. Technol. 34 (2016) 2419 (DOI: 10.1109/ JLT.2016.2535319).

[19] T. Kishi, et al:: "A high-speed LED driver that sweeps out the remaining carriers for visible light communications," J. Lightw. Technol. 32 (2014) 239 (DOI: 10.1109/JLT.2013.2292896).

[20] S. Rajbhandari, et al:: "A multigigabit per second integrated multiple-input multiple-output VLC demonstrator," J. Lightw. Technol. 35 (2017) 4358 (DOI: 10.1109/JLT.2017.2694486). 
[21] E. Fisher, et al.: "A reconfigurable single-photon-counting integrating receiver for optical communications," IEEE J. SolidState Circuits 48 (2013) 1638 (DOI: 10.1109/JSSC.2013.2253222).

[22] R. Y. Chen and Z. Y. Yang: "CMOS transimpedance amplifier for gigabit-per-second optical wireless communications," IEEE Trans. Circuits Syst. II, Exp. Briefs 63 (2016) 418 (DOI: 10.1109/TCSII. 2015.2505264).

[23] X. Li, et al.: "Design and characterization of active matrix LED microdisplays with embedded visible light communication transmitter,” J. Lightw. Technol. 34 (2016) 3449 (DOI: 10.1109/JLT. 2016.2562667).

[24] C.-W. Chow, et al.: "Enhancement of signal performance in LED visible light communications using mobile phone camera," IEEE Photon. J. 7 (2015) 1 (DOI: 10.1109/JPHOT.2015.2476757).

[25] A. V. N. Jalajakumari, et al.: "High speed integrated digital to light converter for short range visible light communication," IEEE Photon. Technol. Lett. 29 (2017) 118 (DOI: 10.1109/LPT.2016. 2624281).

[26] J. J. D. McKendry, et al.: "Visible-light communications using a CMOS-controlled micro-light-emitting-diode array," J. Lightw. Technol. 30 (2012) 61 (DOI: 10.1109/JLT.2011.2175090).

[27] Takai, et al.: "LED and CMOS image sensor based optical wireless communication system for automotive applications," IEEE Photon. J. 5 (2013) 1 (DOI: 10.1109/JPHOT.2013.2277881).

[28] X. Li, et al.: "Design of a 2.2-mW 24-Mb/s CMOS VLC receiver $\mathrm{SoC}$ with ambient light rejection and post-equalization for $\mathrm{Li}-\mathrm{Fi}$ applications," J. Lightw. Technol. 36 (2018) 2366 (DOI: 10.1109/ JLT.2018.2813302).

[29] B. Fahs, et al.: "A 12-m 2.5-Gb/s lighting compatible integrated receiver for OOK visible light communication links," J. Lightw. Technol. 34 (2016) 3768 (DOI: 10.1109/JLT.2016.2587598).

[30] J. Kosman, et al.: "A $500 \mathrm{Mb} / \mathrm{s}-46.1 \mathrm{dBm}$ CMOS SPAD receiver for laser diode visible-light communications," ISSCC Dig. Tech. Papers (2019) 468 (DOI: 10.1109/ISSCC.2019.8662427). 